\title{
Calcul des réseaux mailles de conduite à l'aide d'une calculatrice électronique
}

\section{Pipe network analysis using an electronic computor}

\author{
PAR J. CARTERON \\ INGÉNIEUR AU SERVICE DES ÉTUDES DE RÉSEAUX \\ DIRECTION DES ETUDES ET RECHERGHES DE L'ÉLECTRICITÉ DE FHANCE
}

\begin{abstract}
La méthode exposée dans ce papier n'est qu'un' aspect parficulier d'un'e méthode générale mise au point au service d'études des réseaux de l'Electricité de France et permettant le calcul numérique de réseaux complexes. L'aspect électrique fera l'objet d'une prochaine communication a la Société Francaise des Electriciens. Après un exposé du principe de cette méthode et des bases theoriques sur lesquelles elle repose, un exemple d'application permettra d'en mesurer l'intérêt en même temps qu'il mettra en lumière le changement d'optique que propoque la dispasition des moyens modernes de calcul.
\end{abstract}

\begin{abstract}
The method described in this paper is only at particular application of a general method developed by the Electricite de France network research department which enables complicated networks to be analysed numerically. The electrical aspect will be dealt with in a paper to be presented to the Societe Franctise des Electriciens. After describing the method employed and the theory behind it a practical application gives an indication of its importance and at the same time shows the changed point of view occasioned by the abailability of up to date calculating methods.
\end{abstract}

\section{I. - PROPOSITION PRELIMINAIRE}

Soit un réseau maillé de $b$ branches et $n$ nœuds oì la chute de pression dans la branche $i$ soit de la forme $R_{i} F\left(Q_{i}\right)$ où $Q_{i}$ est le débit dans cette branche et $R_{i}$ un coefficient constant.

Considérons la fonction $\mathrm{U}=\sum_{i=1}^{i=b} \mathrm{R}_{i} \mathrm{G}\left(\mathrm{Q}_{i}\right)$ comme fonction des variables $Q_{j}$, débits des mailles indépendantes au nombre de $m=b-n+1$ où $G$ est défini par $(d \mathbf{G} / d \mathbf{Q})=\mathbf{F}$, $\mathbf{G}$ et $\mathbf{F}$ étant positifs.

Nous supposons donc les débits $Q_{i}$ liés par les équations de nouds et tenant compte des débits injectés, mais non par les équations de maille. La différentielle totale de cette fonction $U$ est identiquement nulle pour les valeurs de $Q_{i}$ vérifiant les équations de maille.

En effet, les dérivées partielles de $U$ sont de la forme :

$$
\frac{\partial \mathrm{U}}{\partial \mathbf{Q}_{i}}=\mathrm{\Sigma} \frac{\partial \mathrm{U}}{\partial \mathbf{Q}_{i}} \frac{\partial Q_{i}}{\partial Q_{i}} \quad \text { où } \quad \frac{\partial Q_{i}}{\partial Q_{i}}=\varepsilon
$$

Si la maille $j$ n'englobe pas la branche $i, \varepsilon=0$. $s=+1$ si la branche $i$ est du sens de la maille $j$. $z=-1$ si la branche $i$ est orientée à l'inverse de la maille $j$.

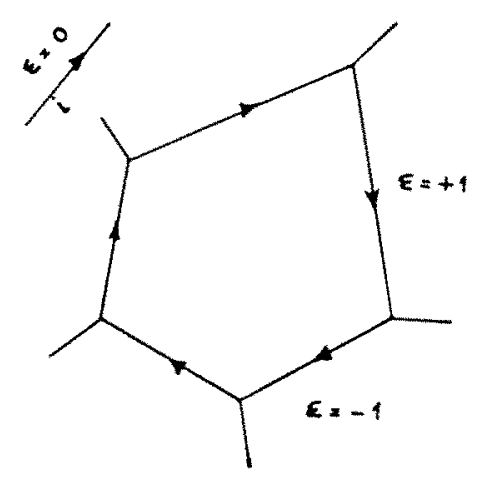

Il en résulte que :

$$
\frac{\partial \mathrm{U}}{\partial Q_{j}}=\mathbf{\Sigma} \varepsilon_{i} \frac{\partial \mathrm{U}}{\partial Q_{i}}=\mathrm{\Sigma} \varepsilon_{i} R_{i} \frac{d \mathrm{G}}{\partial Q_{i}}=\mathbf{\Sigma} \varepsilon_{i} R_{i} F\left(Q_{i}\right)
$$


Lorsque les $\mathrm{Q}_{i}$ vérifient les équations de la maille $j, \frac{\partial \mathbf{U}}{\partial Q_{j}}=0$ donc pour l'ensemble des débits, solution du système, la différentielle totale de $U$ est nulle.

Comme les dérivées secondes :

$$
\frac{\partial^{2} \mathrm{U}}{\partial \mathbf{Q}_{j}^{2}}=\frac{\partial\left(\frac{\partial \mathrm{U}}{\partial \mathbf{Q}_{j}}\right)}{\partial \mathbf{Q}_{i}} \frac{\partial \mathbf{Q}_{i}}{\partial \mathbf{Q}_{j}}=\Sigma \mathbf{R}_{i} \mathbf{F}^{\prime}\left(\mathbf{Q}_{i}\right)
$$

sont positives pour les systèmes physiques où la perte de charge croît avec le débit, cela signifie que $U$ est minimum pour ces solutions et c'est le seul minimum de la surface $Z=U$ dans l'espace des $\mathbf{Q}_{j}$.
Une conséquence immédiate de cette proposition, lorsque $\mathrm{F}(\mathrm{Q})$ est de la forme $k \mathrm{Q}^{\alpha}$, est intéressante à noter à cause de son caractère physique. L'énergie perdue dans un réseau où les injections et extractions de fluide sont inchangées, décroît lorsque l'on ajoute une branche entre deux points quelconques. En effet, U représente cette énergie lorsque les $Q$ sont les solutions du système. L'ensemble des valeurs $Q$ avant addition d'une branche peut être considéré conme un ensemble de débits dans le second réseau (où le débit de la branche ajoutée serait nul) non solution du système mais répondant aux conditions de validité de cette proposition. La valeur de U correspondant à la solution $d u$ second réseau $U_{1}$ est inférieure ou égale à cette valeur $\mathrm{U}_{0}$ qui est elle-même égale à l'énergie perdue dans le premier état du réseau.

\section{II. - PRINCIPE DE LA MÉTHODE}

On part d'un ensemble arbitraire de courants de mailles et l'on corrige ces débits de telle façon que la fonction $U$ décroisse à chaque correction. La proposition précédente nous assure de la convergence de ces valeurs vers la solution du système. Le type de correction adoptée peut être quelconque à la seule condition qu'il assure toujours une décroissance de la fonction U. On peut prendre comme base de calcul la méthode d'Hardy Cross définissant la correction $\Delta Q$ dans le cas où la perte de charge est de la forme $\mathrm{R}_{i} \mathrm{Q}_{i}{ }^{\alpha}$ par la relation de maille :

$$
\Sigma \varepsilon_{i} \mathbf{R}_{i}\left(\mathbf{Q}_{i}+\varepsilon_{i} \Delta \mathbf{Q}\right)^{\alpha}=0
$$

où en négligeant les termes de degré supérieur à $1 \mathrm{du}$ développement $\mathrm{de}\left(1+\frac{\Delta Q}{Q}\right)^{\alpha}$, il vient:

$$
\Delta \mathrm{Q}=-\frac{\Sigma \varepsilon_{i} \mathbf{R}_{i} \mathbf{Q}_{i}{ }^{\alpha}}{\alpha \Sigma \mathbf{R}_{i} \mathbf{Q}_{j}^{\alpha-1}}
$$

On pourrait tout aussi bien appliquer une correction proportionnelle à $-\Sigma \varepsilon_{i} \mathbf{R}_{i} \mathbf{Q}_{i}{ }^{a}$, mais de même signe.

En effet, une correction $\Delta Q$ sur une intensilć de maille provoque une variation :

$$
\begin{aligned}
\Delta \mathrm{U}=\Sigma \mathrm{R}_{i}\left(\mathrm{Q}_{i}\right. & \left.+\varepsilon_{i} \Delta \mathrm{Q}\right)^{\alpha+1}-\Sigma \mathrm{R}_{i} \mathrm{Q}_{i}{ }^{\alpha+1} \\
& =(\alpha+1) \Sigma \varepsilon_{i} \mathrm{R}_{i} \mathrm{Q}_{i}{ }^{\alpha} \Delta \mathrm{Q}+\Delta \mathrm{Q}^{2} \ldots
\end{aligned}
$$

On constate dans les deux cas précédents que $\Delta U$ est négatif tout au moins dans la mesure on $\Delta Q$ est assez faible pour que le premier terme soit prépondérant. Dans l'étude faite, la formule de correction utilisée a été :

$$
\Delta Q=-k \frac{\Sigma \varepsilon_{i} R_{i} Q_{i}{ }^{\alpha}}{\Sigma R_{i} Q_{i}}
$$

où $k$ est positif et a été déterminé expérimentalement de façon à assurer une convergence rapide.

Il avait été prévu de limiter la valeur absolne de $\Delta Q$ au cas où une correction trop forte aurait été une cause de divergence. En fait, cette limitation ne s'est jamais révélée nécessaire. Il est à noter à ce propos que les formules de correction compliquées, telles que celles utilisant, dans le cas où $\alpha=2$, la résolution de l'équation du second degré :

$$
\Sigma \Sigma_{i} R_{i} \Delta Q_{i}{ }^{2}+2 \Sigma R_{i} Q_{i} \Delta Q_{i}+\Sigma \varepsilon_{i} R_{i} Q_{i}{ }^{2}=0
$$

présentent peu d'intẻrêt lorsque l'on utilìse une calculatrice rapide. Il n'est d'ailleurs pas certain que ces formules rigoureuses conduisent au nombre le plus faible d'itération. Une expérience. menée dans la résolution d'un système électrique a montré qu'il y avait un intérêt dans ce cas à multiplier par $4 / 3$ la correction théorique. Cela peut s'expliquer par le fait que si ces formules théoriques provoquent bien la décroissance maximum de U lors d'une correction locale, il peut très bien se faire que les corrections suivantes en soient rendues moins efficaces.

Avant de clore cette partie théorique, il est utile de noter que la méthode de correction des courants n'est pas la seule possible. Il est aussi 
possible de prendre pour inconnues les pressions (ou potentiels) aux nœuds et de les corriger de facon qu'il y ait équilibre des courants. La proposition préliminaire s'étend à ce cas sous la forme :

$$
\mathrm{U}=\mathrm{\Sigma} \frac{\mathrm{P}_{i}^{1+1 / \alpha}}{\mathrm{R}_{i}{ }^{1 / \alpha}}
$$

où $P_{i}$ est la chute de pression dans la branche $i$. La correction à appliquer à la pression est de la forme :

$$
\Delta \mathrm{P}=\frac{\Sigma \eta_{i}\left(\mathbf{P}_{i} / \mathbf{R}_{i}\right)^{1 / \alpha}}{\Sigma\left(\mathbf{P}_{i}(1 / \alpha)-1 / \mathbf{R}_{i}{ }^{1 / \alpha}\right)}
$$

où $\eta_{i}$ tient compte du sens des branches.

Cette méthode n'a pas été appliquée par nous aux réseaux de conduite, mais c'est elle qui a été adoptée et mise en pratique pour la solution des réseaux électriques complexes. Elle présente dans ce cas l'avantage de diminuer le nombre d'inconnues et d'équations, le nombre de nœuds étant inférieur au nombre de mailles du fait que les impédances à la terre créent une maille supplé- mentaire par branche sans augmenter le nombre de nœuds.

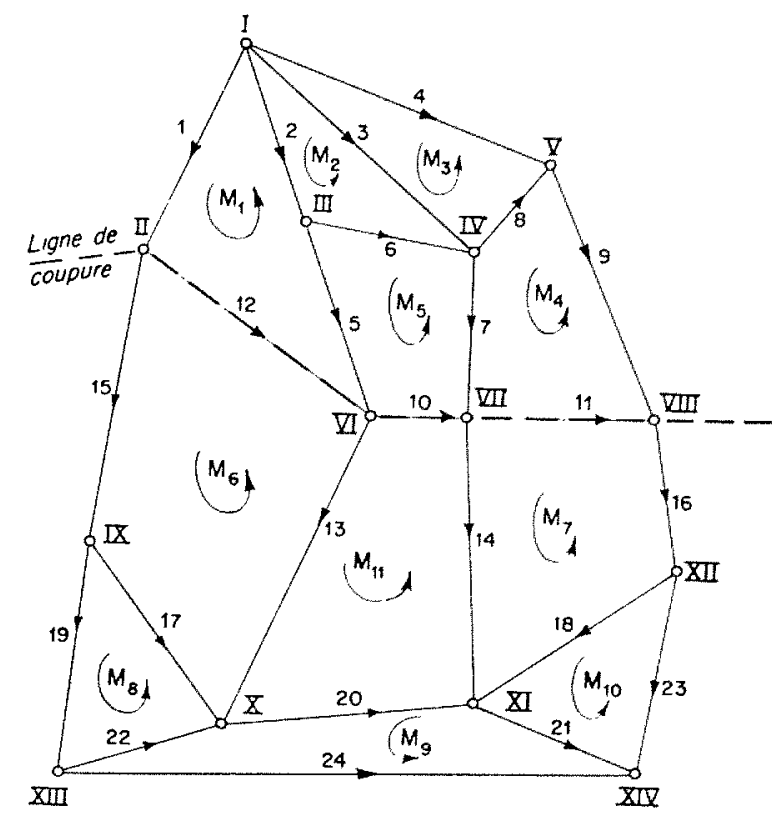

Fig. 1.

Coupure géométrique d'un réseau.

\section{III. - APPLICATION PRATIQUE}

Il n'est pas nécessaire de posséder une calculatrice très puissante pour qu'une telle méthode soit utilement applicable. On peut en particulier traiter des réseaux très complexes avec une calculatrice à capacité de mémoire limitée en des temps raisonnables. Disposant d'une calculatrice du type $\Gamma \mathrm{M}$ de la Société Bull, c'est sur cet outil que nous avons expérimenté cette méthode, et e'est cette application que nous allons décrire.

Cette calculatrice se compose essentiellement d'un groupe de 7 mémoires (dites mémoires de calcul) et de 3 armoires supplémentaires de 8 mémoires que nous appellerons «mémoires de stockage ». Le déroulement du programme peut être contrôlé soit par un groupe de 60 instructions fixes affichées au tableau de connexion, soit par des instructions que nous pouvons introduire par carte.

Le schéma de travail adopté est le suivant:

Les débits des branches sont enregistrés dans les mémoires de stockage. En les définissant par 5 chiffres significatifs et le signe, on peut enregistrer simultanément 48 débits. La séquence de calcul $Q^{\alpha}$ est affichée au tablean de connexion ainsi que deux autres sous-programmes de trans- fert et de contrôle. La succession des cartes introduites en machine est la suivante:

1) Cartes d'introduction des débits initiaux;

2) Groupes de correction de mailles comprenant une carte par branche portant le coefficient $R_{b}$ de la branche et l'adresse du débit de cette branche. Chaque carte déclenche le calcul $\mathrm{R}_{i} \mathrm{Q}_{i}{ }^{\alpha}$ et $\mathrm{R}_{i} \mathrm{Q}_{i}$ et l'ajoute à celui des autres branches de Ia maille. A la fin de ce groupe, une carte d'instructions ordonne le calcul de la correction de maille tandis qu'une dernière earte applique cette correction aux débits considérés.

3) Groupe de cartes transfert et extraction assurant l'impression des résultats d'une itération.

Cette organisation convient à des réseaux ayant au plus 48 branches. Pour des réseanx plus importants, il suffit de découper ce réseau en zones telles que les calculs d'une zone n'impliquent que l'enregistrement simultané de 48 débits. L'organisation précédente se retrouve pour chaque zone. On perfore à l'extraction de fin de zone les débits corrigés qui seront réintroduits à l'itération suivante. Le principe de la coupure est 


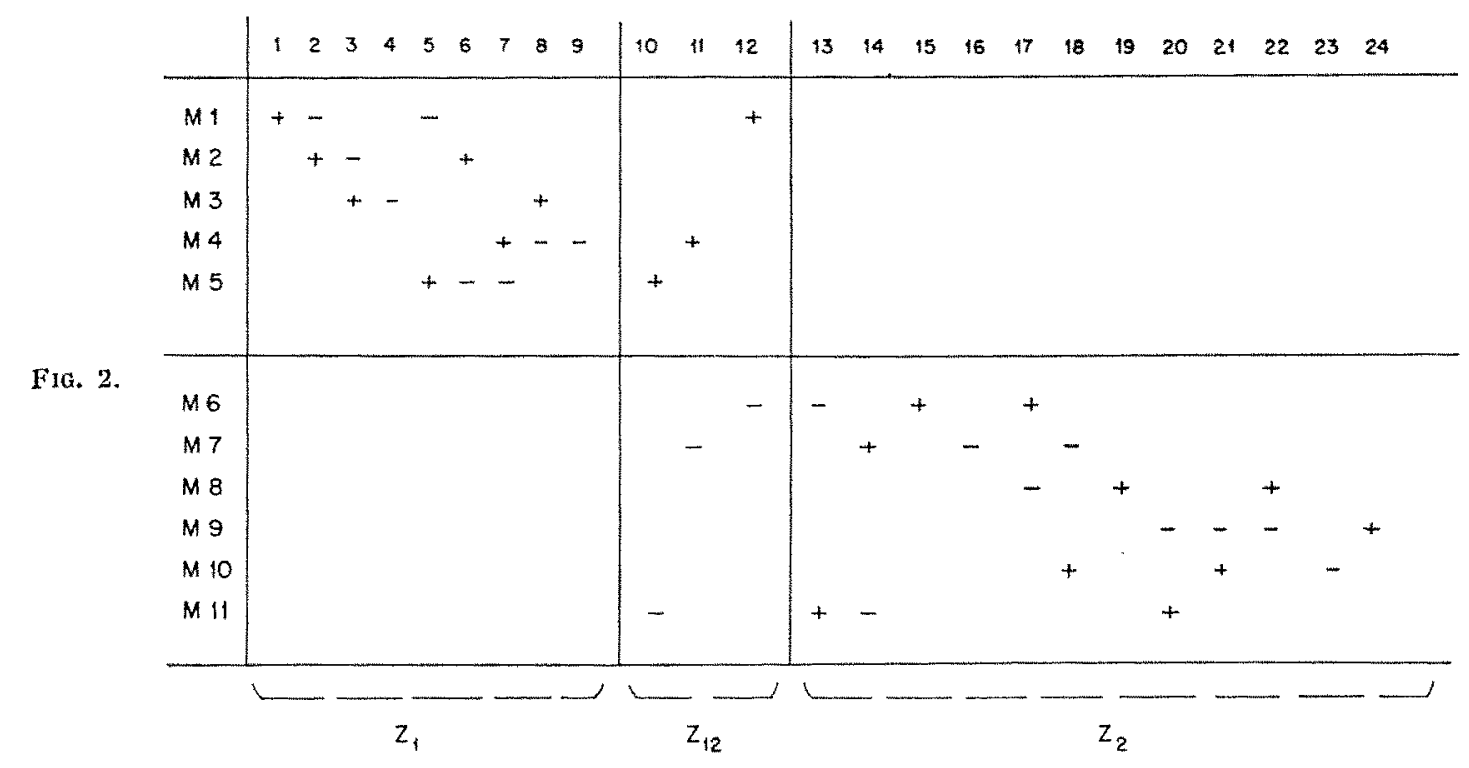

explicitee sur les figures 1 et 2 et la succession des cartes sur la figure 3 .

Il faut bien noter que cette coupure n'interfère en rien sur le déroulement des opérations de calcul. C'est uniquement un artifice permettant de pallier le manque de capacité de la machine dont nous disposions. Cette extraction intermédiaire nous a cependant imposés quelques modifications au schéma de base :

a) Nécessitẻ d'un programme de contrôle détectant les éventuelles erreurs de perforation;

b) Répétition des itérations à l'intérieur d'une même zone pour diminuer ces extractions;

c) Enregistrement en calculatrice du programme de transfert.

Une autre organisation consisterait à n'enregistrer que les débits de maille et à calculer les débits de branches par somme algébrique. Elle aurait l'avantage de nécessiter l'enregistrement d'un nombre réduit de grandeurs : le nombre de mailles indépendantes étant très inférieur au nombre de branches. Il a été adopté pour les réseaux électriques et pourrait avantageusement être employé ici.

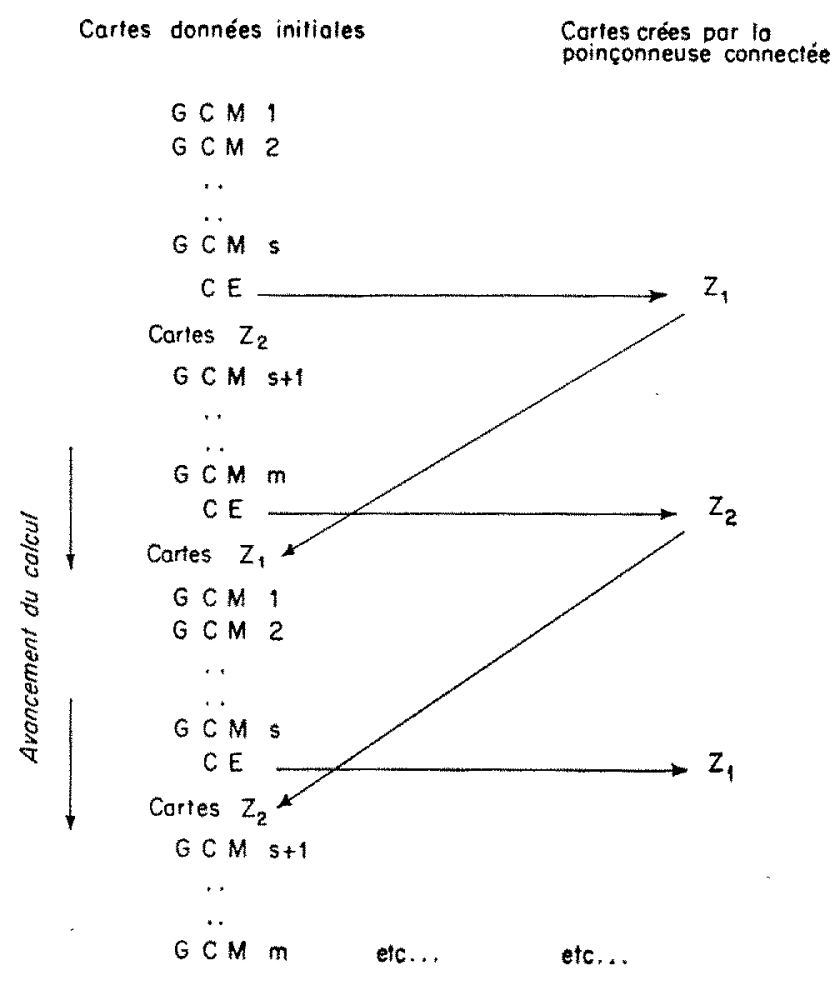

Fig. 3.

\section{IV. - RESULTATS}

Notre expérience est limitée, la mise au point élant récente. Le plus gros exemple traité concerne un réseau de 75 branches et 29 mailles représenté figure 4 .
A la $22^{\circ}$ itération, le dernier chiffre significatif était fixé. Puisque le nombre de cartes nécessaires par itération est de l'ordre de $2(b+m)$, soit ici environ 200 cartes, le temps théorique 


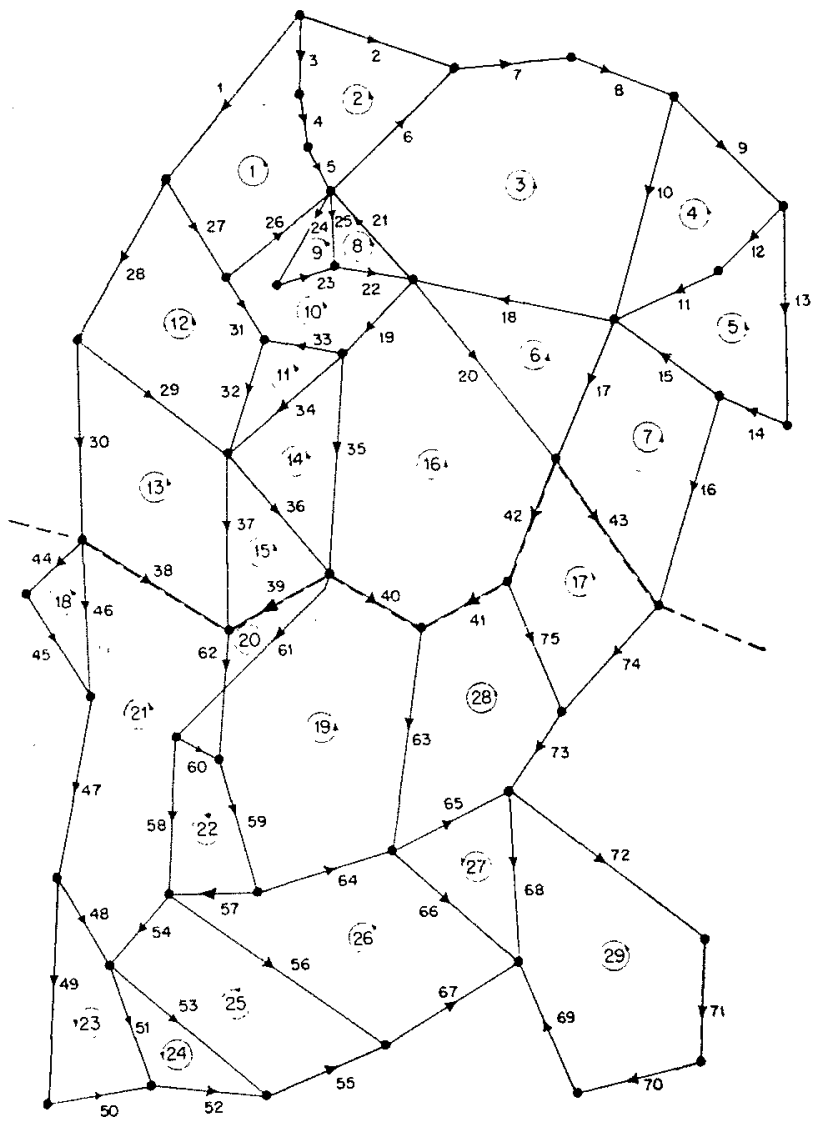

FIi. 4.

de résolution est de :

$$
\frac{200 \times 22}{150}
$$

soit une demi-heure, la machine traitant 150 cartes par ninute. En tenant compte des manipulations et des contrôles, on peut compter une heure environ, au bout de laquelle on obtient non seulement les débits mais aussi les chutes de pression sur toutes les branches et, si on le désire, l'énergie dépensée.

En face de ce temps bref, quel est le temps de préparation?

A ce sujet, il ne faut pas confondre le travail de programmation, de mise au point des connexions et d'organisation et le travail spécifique à un calcul donné. Le premier est grand, mais si l'organisation est bonne, le second est très bref. Si l'on veut établir une comparaison avec un analogue, le premier serait à comparer à l'étude de l'analogue lui-même et le second à l'affichage d'un problème. Sous cet angle, la méthode arithmétique est bien plus légère que l'autre et très certainement plus économique pour une entreprise à problèmes multiples. Que fautil faire en fait devant un problème nouveau? D'abord construire sa matrice de connexité (fig. 2) qui représente la topologie du résean. Puis faire un état ordonné des branches et faire perforer sur des cartes spécialement préparées dans un fichier les valeurs des coefficients $R_{i}$ et des débits initiaux. C'est un travail de quelques heures exécutables par un personnel soigneux mais sans connaissances spéciales. Si l'on veut modifier ensuite ce réseau, ces modifications se ramènent à l'échange de quelques cartes, opération simple et rapide.

Avant de conclure, deux remarques importantes sont à faire :

a) Le nombre d'itérations dépend très peu du système initial de valeurs. L'ordre de grandeur se fixe en tout état de cause dans les 10 premières itérations. Il est donc tout à fait inutile de chercher une répartition astucieuse au départ: c'est perdre son temps;

b) Certains auteurs insistent sur le fait qu'il vaut mieux itérer dans un ordre ou un autre selon certains critères. Ces considérations perdent tout intérêt ici. Il vaut mieux garder une organisation rigide qui simplifie la mise au point. S’il en résulte quelques itérations de plus, cela n'a guìre d'importance.

Ces remarques mettent particulièrement en évidence le changement de point de vue qu'entraine l'usage de calculatrices rapides. Nous touchons ici du doigt le début d'une révolution qui atteindra peu à peu toutes les branches de la technique. Il y a une mathématique des grands ensembles d'équations qui se développe sur des voies à peine explorées. L'introduction des machines à grande capacité accentuera cette évolution que les premières calculatrices électroniques ont amorcée. Il n'en est que plus souhaitable que les ingénieurs de toute technique soient informés des moyens qui leur sont offerts.

\section{DISCUSSION}

Président: M. Remenieras

M. le Président remercie M. Carteron et souligne it grand intérêt des machines électroniques pour la solution rapide de nombreux problèmes coinportant des systèmes d'équations linéaires simultanées à $n$ inconnues: dans certains domaines, comme celui des propa- gations d'ondes et, particulièrement de la marée, les méthodes graphiques cèdent le pas à ces méthodes qui paraissent intellectuellement moins élégantes mais sont en fait plus rapides.

Sur la demande de M. Guéneav, qui regrette tout 
d'abord l'absence de M. Dubis, M. Canteron précise que dans la méthode Hardy-Cross la valeur du coefficient in qui a été jugée la meilleure daus le calcul exposé est compris entre 1 et $4 / 3$; ce nombre n'est pas une valeur critique, mais il résulte d'un essai qui a comporté 45 itérations en prenant $\mathrm{K}=1$ et seulement 22 en prenant $\mathbf{K}=4 / 3$.

M. GUÉnEAU indique, toutefois, que, dans un réscau à deux canalisations de même résistance, pour lequel on partirait d'une répartition inégale des débits entre les deux branches, la méthode Hardy-Cross donne, avec $\mathrm{K}=1$, la solution exacte dès le premier calcul, alors que, en prenant $K=4 / 3$, il faudrait six on sept tours pour réduire l'erreur au $1 / 1.000$ de l'erreur initiale.

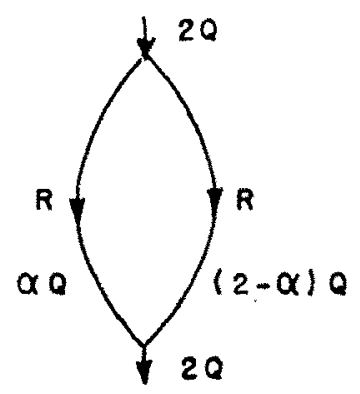

M. Cantenon fait remarquer que la valeur optimum de $K$ est. celle qui est globalement la meilleure et qu'il est normal de trouver des cas particuliers ou $I^{K}=1$ soil loptimum.

W. GuÉnEAu indique ensuite que les calculs faits à la Compagnie Générale des Eaux depuis 1947 avec $K=1$ donnent des valeurs trop faibles des corrections successives, mais que, étant donné la complexité et la diversité des réseaux, il a été impossible de trouver une systématisation $d u$ coefficient de majoration $K$.

M. Canteron pense qu'avec un peu d'expérience, que pourra sans doute donner le programme d'essais sur réseaux électriques actuellement en cours, on arrivera à la conclusion que 1,20 est probablement, en moyenne, le coefficient qui convient le mieux.

M. GUENEAU indique qu'un essai fait avec $K=4 / 3$ dans un réseau simple lui a donné une bonne convergence, mais que ce coefficient devrait être en fonction de plusicurs éléments tels que la somme des résistances de différentes canalisations composant une même maille, le degré d'itération...

Il estime, d'autre part, que les principes de la correction en cascade et du choix des mailles de moindre résistance, donnés par M. Durin dans son article de «la Ilonille Blanche $»$, en 1947, conservent leur valeur.

M. Gúnea ajoute que la C.G.E. fait depuis quelgue temps déjà et antérieurement à E.D.F. des calculs de réseaux sur machine électronique. La méthode employée sur le C.P.C. de la compagnie I.B.M. (calculateur à programme par Cartes, comportant 24 mémoires) consiste à perforer sur cartes les débits initiaux dans chaque branche, à calculer ensuite les débits correctifs daus chaque maille et à envoyer en mémoire la somme algébrique des corrections successives de chaque maille: ce procédé implique la réservation de 4 ou 5 mémoires pour les calculs intermédiaires et permet done le calcul de réseaux comportant une vingtaine de mailles et un nombre quelconque de canalisations. Une nouvelle machine I.B.M., \&lordinateur 650 », à 2.000 mémoires, donnera prochainement à la C.G.E. des possibilités de calcul pratiquement illimitées, sans ancune coupure du réseau.

M. Canteron confirme l'intérêt de mettre en mémoires les éléments correctifs sur les malles platôt que sur les branches, ainsi que cela lui est apparu au cours de calculs de réseaux électriques. D'autre part, M. Cantenon estime que l'intérêt de la question est de montrer que, même avec une machine plus simple, on peut travailler raisonnablement sans limitation stricte.

M. GUÉNEAU attire l'attention sur l'intérêt des méthodes qui permettent dacerôtre la rapidité de la convergence et donc de réduire le temps d'immobilisation de la machine, ce qui, dans le cas d'une machine louée, représente une économie sensible, mème si lon tient compte du léger supplément de travail préparatoire que cela comporte.

M. Guḱnsay et M. Carteron sont d'allleurs d'aceord pour estimer que l'objection d'un long temps de préparaliou, faile, ea général, aux calculs électroniques, ne résiste pas à l'examen, si l'on veut bien mettro en balance le temps de méditalion, tout a fait comparable, que nécessiterait la préparation d'un réseau de résislanees dans la méthode analogique. De plus, la machino électronique fait n'importe quel calcul (calcul de réseau électronique ou de comptabilité), elle ne nécessite aucun travail intermédiaire et donne, sous forme d'états imprimés des résultats directement exploitables, avec telle précision que l'on désire. Enfin, les calculs relalifts aux conduites ajoutées paraissent aussi simples que par la méthode analogique.

Sur la demande de M. de Brem, M. Carteron précise que, une lois étudiées les méthodes à mettre en ceuvre pour le calcul du réseau, les opérations successives nécessitèes par le calcul des coefficients électriques absorbent au total 1 journéc environ, pour un réseau de l'ordre de 75 mailles.

Sur la demande de M. le Président, M. Carteron indique que la substitution de pertes de charge quadratiques, dans le domaine hydraulique aux chutes de tension linéaires en électricité n'entraine aucune complication : il suffit d'introduire un programme avec la puissance 2 au lieu de la puissance 1.

Sur la demande de M. Guéneau, M. Cantenon précise que les débits sont calculés à la machine électronique an millième de litre par seconde.

Sur la demande de M. Hupner, M. Carteron indique que, dans le domaine électrotechnique, la comparaison des tables de calcul analogique avec les machines électroniques est encore trop récente pour permettre un jugement circonstancié; il semble que le résultat de cette comparaison dépendra surtout de la nature du problème: en particulier, dans un problème des pertes do charge dans un réseau électrique, la méthode électronique doit prévaloir par sa précision.

M. le Président conclut sur le plan général en remarquant que la machine électronique peut traiter des problèmes très différents les uns des autres, alors qu'un appareil analogique ne peut guère traiter que les problèmes pour lesquels il a été conçu.

M. HUpNer envisage d'autre part l'application de la machine électronique au calcul de la résistance mécanique des barrages qui, dans la méthode Trial-Load conduit à la résolution d'un système de $n$ équations linéatres à $n$ inconnues : sur sa demande, M. Carteron indique que la machine faisant l'objet de son expose peut résoudre un système de 26 équations et que cette operation néessiterait le temps de perforation de 400 ou 500 cartes.

M. Hupner ajoute que, quand on fait ces calculs avec moins d'équations par les méthodes classiques, on se heurte souvent, au point de vue de la précision, à la difficultê suivante. Il $y$ a des cas où, tel que le calcul se conduit à un déterminant final qui apparait comme in différence faible de nombres assez grands qui ne sont pas très bien connus; le résultat final manque de précision 
a cause de cela. C'est le cas de certaines abaques étrangères et M. Mŕriaux en appliquant les mêmes formules, mais en s'y prenant autrement, a établi des abaques beancoup plus précises. Est-ce que, avec la machine, on risque d’avoir ces difficultés?

M. Carteron pense que, lorsqu'un système est voisin de l'indétermination, le calcul électronique doit aboutir ì la même imprécision.

M. Schlumberaen pense que ce cas est peut-être celui d'une matrice vicieuse, qui, dans le cas d'un système de deux équations à deux inconnues, se traduit par deux droites presque parallèles, dont le point d'intersection serait mal déterminé. Mais pour un système de la forme $a_{1} \quad x_{1}+a_{3} x_{2}+\ldots \ldots+a_{n} x_{n}=b_{1}$ etc. il est très difficile de voir dans quelle mesure ce système est vicieux.
D'ailleurs, il se peut que, si l'on a résolu le problème posé, on arrive à une solution qui n'est pas valable.

M. Le Président indique que, dans le cas particulier d'un système de plus de 100 équations simultanées qu'il a rencontré, la vérification préalable de l'éventuelle indétermination de celui-ci aurait con̂té aussi cher que sa r'ćsolution.

M. Carteron remarque que les méthodes analogiques comportent souvent de petites erreurs qui se cumulent et peuvent atteindre un pourcentage très important en fait, ainsi qu'il a pu s'en rendre compte en comparant les résolutions d'un système avec une « table * analosique et avec une calculatrice électronique. Par consé. quent, dans des cas de ce genre, la précision élevée des calculatrices numériques n'est pas inntile.

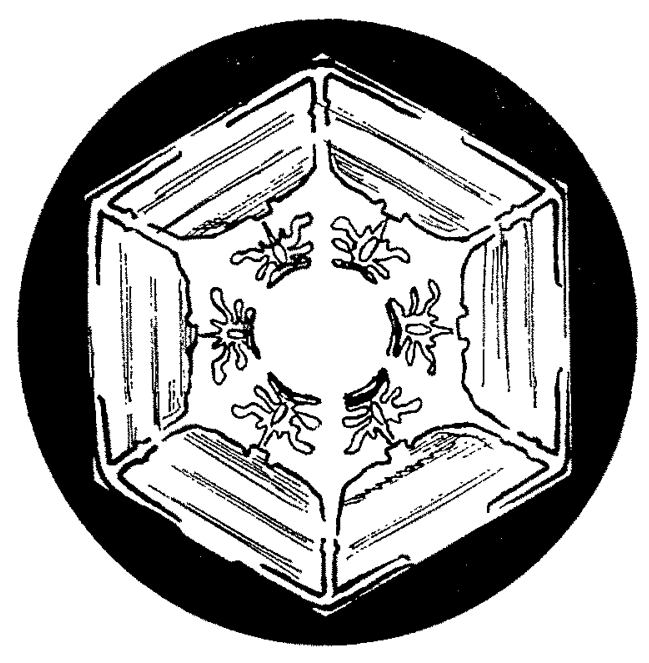

